Relations industrielles

Industrial Relations

\title{
L'organisation du travail et ses fonctions nouvelles, Paris, La Documentation Française, 1977, 292 pp.
}

\section{Gilles Dussault}

Volume 33, numéro 1, 1978

URI : https://id.erudit.org/iderudit/028852ar

DOI : https://doi.org/10.7202/028852ar

Aller au sommaire du numéro

Éditeur(s)

Département des relations industrielles de l'Université Laval

ISSN

0034-379X (imprimé)

1703-8138 (numérique)

Découvrir la revue

Citer ce compte rendu

Dussault, G. (1978). Compte rendu de [L'organisation du travail et ses fonctions nouvelles, Paris, La Documentation Française, 1977, 292 pp.] Relations industrielles / Industrial Relations, 33(1), 149-151.

https://doi.org/10.7202/028852ar

Tous droits réservés @ C Département des relations industrielles de l'Université Laval, 1978
Ce document est protégé par la loi sur le droit d'auteur. L'utilisation des services d'Érudit (y compris la reproduction) est assujettie à sa politique d'utilisation que vous pouvez consulter en ligne.

https://apropos.erudit.org/fr/usagers/politique-dutilisation/ 
l'état du marché du travail au Québec. Les études approfondies que les auteurs nous promettent sont attendues avec impatience.

\section{Claude RONDEAU}

\section{Université Laval}

\section{L'organisation du travail et ses formes nouvelles, Paris, La Documentation Française, 1977, 292 pp.}

La littérature sur les thèmes de l'humanisation du travail, du réaménagement des tâches, de la participation des travailleurs à la gestion des entreprises se multiplie depuis une dizaine d'années. Malgré l'abondance de documentation, peu d'ouvrages ont la qualité d'offrir en même temps au lecteur une excellente introduction à ces questions complexes et une synthèse rigoureuse des connaissances disponibles à leur sujet.

C'était l'objectif du Centre d'études et de recherches sur les qualifications qui publie cet ouvrage collectif et pour qui "le souci d'engager des actions efficaces autant que celui d'améliorer la connaissance font ressentir le besoin de mesurer jusqu'à quel point l'état où se trouvent les travaux scientifiques permet de maîtriser les problèmes à traiter», selon les mots de son directeur, M. Gabriel Ducray.

L'ouvrage propose neuf (9) articles présentés par A. d'Iribarne (pp. 9-26) ainsi qu'une bibliographie qui rassemble l'essentiel de la littérature scientifique française sur les formes nouvelles d'organisation du travail.

On aurait pu regrouper la majorité des articles autour des deux thèmes: celui de la division du travail d'abord et de ses déterminants, avec les contributions de René Cessieux ( Approche historique et critique de la division du travail », pp. 2772), Maurice Chevalier ("Conditions de détermination des systèmes socio-techniques ", pp. 73-92) et Daniel Montjardet ( $« \mathrm{La}$ variable "technologie» dans les études d'organisation. Bilan critique», pp. 93122). L'autre thème est celui des formes nouvelles d'organisation du travail et leur mise en place; nous y incluons les articles de Jean Ruffier ( Les nouvelles formes d'organisation du travail dans l'industrie française», pp. 123-154), Georges Trepo, ( Stratégies d'introduction des nouvelles formes d'organisation du travail», pp. 155-170), Renaud Sainsaulieu ( «Sur les traces de fonctionnement collectif dans les organisations », pp. 171-194) et enfin de Béatrice Belbenoît et André Zilberberg ( Projets autogestionnaires, organisation des entreprises et équilibre économique », pp. 195-218). Deux autres textes complètent l'ouvrage, un traitant des apports de l'ergonomie à l'amélioration des conditions de travail, (Antoine Laville, «Ergonomie et conditions de travail», pp. 219-234) et l'autre faisant le bilan des études et des législations en matière d'organisation du travail en France depuis 1968 (Françoise Meylan, «Les conditions de travail à travers les études entreprises à l'initiative des pouvoirs publics: l'évolution récente de la législation et de la négociation collective», pp. 235-278).

L'intérêt que nous semblent présenter ces articles, c'est de soulever un ensemble de questions parmi les plus fondamentales que pose l'évolution récente de l'organisation du travail dans les sociétés industrielles. L'évolution des formes de travail vers un travail plus riche de significations et plus responsable s'inscritelle dans une continuité historique ou marque-t-elle une rupture radicale? De quelles marges de liberté dispose-t-on pour modifier l'organisation du travail ? À quelles conditions, des changements à ce niveau peuvent-ils être diffusés? A-t-on épuisé les ressources de l'organisation du travail artisanal ou coopératif? Quelles sont les possibilités d'implantation des projets autogestionnaires dans les économies occidentales?

René Cessieux cherche à déterminer à quels principes répond le système de découpage et de combinaison des tâches dans une société et à identifier quelles sont les formes historiques successives de la division du travail ainsi que les facteurs qui l'ont boulversée. Pour éclairer ces interrogations, il propose un survol de la pensée économique et sociologique (Adam Smith, Marx, Durkheim, Schmoller et Bucher de l'école historique alle- 
mande) ainsi qu'une critique serrée des thèses maintenant bien connues de Stephen Marglin et André Gorz (André Goz (éd.), Critique de la division du travail, Paris, le Seuil, 1973). Pour ces derniers la division du travail aurait pour fonction essentielle, non celle d'élever la productivité, mais de "reproduire les rapports de domination du capital sur le travail»; «le capitaliste divise pour règner». Cessieux reproche à cette thèse de confondre les deux volets du procès du travail, soit l'accumulation du capital et la division du travail. En cherchant ce qui est à l'origine de la division du travail et de la hiérarchie capitaliste, Marglin n'envisage que deux réponses mutuellement exclusives: «soit le souci de diviser pour règner, soit (...) la recherche de l'efficacité ». Cessieux refuse ce choix et démontre qu'historiquement ces deux objectifs se sont combinés et qu'il n'y a pas de cause unique à l'évolution de la division du travail, ce qui est lourd d'implications lorsqu'on propose des stratégies de transformation de la division du travail.

Maurice Chevalier demande si les formes nouvelles d'organisation du travail ne seraient pas «en fin de compte, un complément des méthodes traditionnelles (le «taylorisme») qui ont atteint leurs limites et ne peuvent être développées que dans ce sens» (p. 75). Par exemple, les coûts des matériaux et outils n'exigent-ils pas que l'on suscite l'engagement de l'individu dans la réalisation de sa tâche? D'où l'acceptation par de plus en plus d'entreprises de s'ouvrir à l'enrichissement des tâches et à la responsabilisation des groupes de travail.

Daniel Montjardet examine, de son côté, la question de la détermination des structures des organisations par la technologie. À partir d'un bilan critique des travaux des groupes formés autour de Joan Woodward et de David Hickson et ses collègues, il conclut que "puisque c'est bien le produit qui est le support physique de la marchandise, il est théoriquement insoutenable de penser que technologie et organisation puissent être radicalement autonomes, il l'est tout autant de poser que l'organisation puisse être étroitement asservie à des exigences techniques absolues alors que le produit n'a pour elle de sens qu'en terme de marchandise. c'est-à-dire de marché et de coût de production. C'est entre ces deux bornes que la relation entre technologie et organisation prend sens, c'est-à-dire qu'elle n'est pas interprétable sans prise en compte notamment de la situation [plus ou moins concurentielle] sur le marché des produits, des exigences donc que les stratégies commerciales imposent à la production, des choix d'investissements qui en découlent, etc. tous éléments désespérément absents de presque toutes les recherches examinées » (p. 118).

Quant à Jean Ruffier, il propose une analyse des expériences françaises de réorganisation du travail - il estime le nombre des postes enrichis entre 10,000 et 35,000 - à partir d'une typologie des façons d'introduire le changement; il distingue les modèles expérital, participatif et négociateur. Pour lui il est encore tôt pour évaluer l'impact de ces expériences, mais il semble bien que l'organisation du travail est en train de devenir un enjeu politique.

La contribution de Renaud Sainsaulieu à cet ouvrage est particulièrement intéressante; elle rappelle au lecteur que l'organisation du travail dans les sociétés industrielles n'est pas que taylorierıne. Tout un réseau d'entreprises - de petite taille le plus souvent - utilise des formules de fonctionnement collectif. Sainsaulieu a observé des entreprises artisanales, coopératives ou encore nettement participatives et en retient comme caractéristique particulière l'existence d'un réseau de pouvoirs où chacun des membres de l'organisation possède une marge de négociation soit en raison de son expertise, de sa position stratégique dans l'entreprise, soit en vertu d'une volonté collective de partager le pouvoir.

Sainsaulieu rappelle, s'il est besoin, qu'il y a loin de la définition d'une structure participative à sa mise place dans la réalité quotidienne. S'il est relativement aisé de modifier des structures hiérarchiques, il est à retenir que les individus, eux, ne changent pas sans passer par un processus complexe d'adaptation. " $\mathrm{Ce}$ n'est pas parce qu'on propose des structures coopératives ou de partage officiel du pouvoir que les acteurs perdent leur 
culture, leurs motivations complexes et leurs capacités stratégiques. Le problème est alors de voir à quelles conditions de telles structures peuvent s'apprendre et selon quels processus d'alliances elles se mettent en place et se maintiennent en vie» (p. 181). C'est une réflexion à méditer en examinant les «projets autogestionnaires" de la C.F.D.T., du P.S.U. ou du P.S. que présentent B. Belbenoît et $A$. Zilbergerg ou en relisant les comptesrendus des nombreux rapports commandés par les gouvernements français depuis dix ans dont F. Meylan fait la nomenclature.

Cet ouvrage doit être lu par ceux qui veulent faire le point sur les possibilités d'agir sur l'aménagement des tâches parce qu'il replace dans leur contexte les expériences en cours et qu'il rappelle jusqu'à quel point le chemin de l'humanisation du travail industriel est long et difficile.

Gilles DUSSAULT

Université Laval

Canadian Labour Standards in Law, Agreement, and Practice by Paul Malles, Ottawa, Economic Council of Canada, 1976, 176 pp.

Cet ouvrage constitue une synthèse des principales conditions de travail régissant les employés, dont une attention particulière apportée aux règles relatives à l'accès au marché du travail, à la protection du lien d'emploi et à la cessation de la relation du travail.

On notera un premier chapitre devenu traditionnel dans ce genre d'ouvrage: "The Development of Labour Standards in Canada». L'auteur y présente une description de l'évolution des normes du travail et de son interaction avec la négociation collective. Le principal mérite de ce chapitre est d'avoir su mettre en relief que certaines catégories de travailleurs se voient encore de nos jours refuser l'accès aux conditions générales et minimales de travail, tels par exemple les domestiques, les employés de fermes, les jeunes travailleurs en certaines occasions, etc.

Un second chapitre intitulé «The Institutional Framework for Establishing and
Enforcing Labour Standards» présente une étude bien structurée des organismes impliqués dans l'établissement, le contrôle et la mise à jour des conditions de travail. Il est intéressant de constater que la Nouvelle-Écosse et l'Alberta ont su conserver leurs pièces législatives intégrées sur les normes de travail (1973); il en va de même avec le Code canadien (1972). Par ailleurs, exception faite de l'Ontario et du Canada, les autres provinces canadiennes continuent à maintenir en place des organismes particuliers pour s'occuper de l'administration des normes de travail, organismes en principe indépendants des ministères.

L'étude des conventions collectives constitue l'objet du troisième chapitre: «Labour Standards Under Collective Agreement». Une analyse de certains types de clauses mérite une lecture attentive. Il s'agit en fait d'une synthèse portant sur les sujets suivants: (i) Union Security; (a) Union Membership as a Condition of Employment; (b) Union Dues Check-off; (ii) Management Rights; (iii) Employee Security; (a) Seniority; (b) Hiking and Firing; (c) Technological Change ;(d) Protection of Special Groups; (iv) Health and Welfare Plans. Le cinquième chapitre se situe dans le prolongement des troisième et quatrième en ce qu'il constitue une étude intitulée "Work and Rest». L'auteur axe son analyse avant tout sur les normes minimales d'origine étatique situant à l'occasion l'apport des conventions collectives.

Enfin, l'auteur traite dans un sixième chapitre des difficultés rencontrées par les «professional worker» («The Special Case of Professionnel Workers»), plus particulièrement en matière d'organisation syndicale.

L'ouvrage est complété par des propos de synthèse («Summary and Conclusions») et par une série de tableaux sur les différentes normes de travail.

Sommairement, on peut dire de cet ouvrage qu'il constitue une œuvre de synthèse assez remarquable sur les coordonnées juridiques et institutionnelles concernant l'emploi.

Rodrigue BLOUIN

Université Laval 